\title{
Evolution in Revolution
}

\author{
Denis Noble
}

\author{
The Demon in the Machine: How Hidden Webs of \\ Information Are Solving the Mystery of Life \\ by Paul Davies \\ Penguin, 272 pp., $\$ 25.00$.
}

$\mathrm{P}$ HYSICAL SCIENTISTS and mathematicians have made important contributions to the foundations of biology and the theory of evolution. There is, as well, a profound historical connection between Erwin Schrödinger's What Is Life?, which was delivered as a series of lectures in 1944, and molecular biology; it is a connection that both Francis Crick and James Watson acknowledged. ${ }^{1}$ Schrödinger predicted that the genetic material within the cell would be an aperiodic crystal, one that expressed what he called a code script. ${ }^{2}$ The code script, Watson and Crick discovered in 1952, is DNA. A long sequence of nucleotide bases, DNA functions within the cell as the template governing the formation of proteins. These are, in turn, comprised of amino acids, and so belong to an entirely different cellular kingdom. The mapping from DNA to the proteins, Crick argued, went in one direction. DNA governed the formation of proteins from various amino acids, and never the reverse.

This is the central dogma of molecular biology.

If the connection between What Is Life? and the central dogma is direct because it is historical, the central dogma is now known to be incorrect. ${ }^{3}$ Much of the evidence in favor of the demotion of the central dogma is brilliantly expounded by Paul Davies in The Demon in the Machine. Schrödinger brought crystallography to bear on the puzzles of stability and heredity; Davies brings information theory to bear on the same puzzles. In doing so, he joins the distinguished company of physical scientists who have contributed to fundamental biology.

Although I have reservations about the concept of information in biology, I am in agreement with many of Davies's conclusions. In discussing cell division, he points out that the genome is entirely passive. It is the cell that does the dividing. DNA is as much acted upon as acting. If so, the conventional framework of biological theory is misleading. "What is still a mystery," Davies writes, is the biological equivalent of the supervisory unit that determines when instructions need to switch to become passive data. There is no obvious component in a cell, no special organelle that serves as "the strategic planner" to tell the cell how to regard DNA (as software or hardware) moment by moment. The decision to replicate ... is not localized in one place. ${ }^{4}$

The book's frequent references to top-down causation are welcome. In 2011, George Ellis organized an important meeting that brought this topic to the fore. ${ }^{5}$ At the meeting, I argued that there is no privileged level of causation in biology. ${ }^{6}$ This has been clearly shown in the mathematical modeling of biological networks. I have been arguing for this principle ever since. Davies, it is satisfactory to recount, expresses the same idea, but by a different metaphor.

In the nineteenth century, James Clerk Maxwell imagined a tiny creature that could open and close a miniscule aperture between two compartments, one warmer and one cooler, and could distinguish between fast- and slow-moving molecules. This creature, he suggested, could preferentially let the few fast ones from the cool compartment pass through to the warm one. As a result, the warm compartment would become even hotter, while the cool one would become even colder. Such a demon could reverse the normal flow of heat. Maxwell's demon appeared to reverse the laws of thermodynamics.

Davies shows that organisms have such molecular demons working away throughout the body. They can do this, of course, because they are open systems, continually exchanging matter and energy with their environment. Any energy used by the molecular demons comes from the environment with which living systems are in communion. Charles Darwin saw this very clearly as well. "In my opinion," he wrote in 1876, "the greatest error which I have committed, has not been allowing sufficient weight to the direct action of the environment, i.e. food, climate, etc., independently of natural selection."

How do nanoscale molecular motors, Davies asks, achieve their effects? Living cells, he answers, are highly structured. Tiny filaments form roadways along which 
messages can be sent from one part, such as a surface receptor sensing food or danger, to another part, such as the DNA stored in the nucleus. The process can operate with pinpoint accuracy. The immune system uses it to good effect when, by way of such targeted DNA changes, it generates antibodies to neutralize unfamiliar invading viruses or bacteria. Cells can detect serious danger and effectively change the arrangement or composition of their DNA. These processes are in some way guided because without such guidance, they could not produce the desired result.

A word is needed for this. Fortunately, one is on hand, which has been in use since the time of Aristotle. It is teleology. The process is rather like shuffling a pack of cards. By chance, many different new arrangements will occur. The operative unit-whether a multicellular organism or a single-cell organism-then selects the arrangement best suited to cope with the environmental challenge. What holds for the cell, holds as well for evolution itself. Davies refers to biologists who have done important work in this regard, among them Kevin Laland, who has worked on niche construction, ${ }^{8}$ and Patrick Bateson, who has studied what he calls adaptability drivers. ${ }^{9}$ These arguments serve to weaken the common neo-Darwinian assumption that evolution is completely blind, and they suggest, if they do not imply, that life is not simply an unlikely occurrence in a universe without purpose.

Organisms steer their own evolutionary course, a process made possible by Davies's molecular demons. As he makes clear, this is not a sufficient explanation. What enables organisms to control their genes and other molecular mechanisms? Not the demons themselves. They do as they must. Davies calls for new laws of physics that must control, or make possible, the networks of life. A gap needs to be filled to connect high-level decisions with low-level molecular machinery:

The way the laws of physics are currently conceived leads to a stratification of physical systems with the laws of physics at the bottom conceptual level and emergent laws stacked above them. There is no coupling between levels. When it comes to living systems, this stratification is a poor fit because, in biology, there often is coupling between levels, between processes on many scales of size and complexity: causation can be both bottom-up (from genes to organisms) and top-down (from organisms to genes)..$^{10}$

It is crucial, in this regard, to distinguish between different forms of causation. Top-down causation represents a constraint, or boundary condition, within which molecules follow the usual laws of molecular interaction. It is a view of causation suggested by the theory of ordinary differential equations. Ellis has made significant advances pursuing this idea in his recent book, How Can Physics Underlie the Mind? ${ }^{11}$ I am following a proposal made at the Royal Society and British Academy 2016 meeting on New
Trends in Evolutionary Biology that provides a second sense of causation, one linked to the classical theory of stochastic differential equations. The modern synthesis gives too small a role to chance at the molecular level. ${ }^{12}$ There is far more potential stochastic variation in the genome than biologists imagine. The actual error rate when DNA is being copied is around 1 in $10^{4}$ bases-a figure a million times higher than previously assumed.13 Davies describes a process in which proofreading demons come in to diligently compare one strand of copied DNA with the other to discover where the errors occur, and then to correct them. Imagine a proofreader receiving a book draft with so many errors. It would amount to an error on almost every page. At 100 pages per book, the demons would clean up all the errors in 10,000 such books. No human proofreader could be that accurate. Imagine now that the organism can selectively vary the error-correction rate. By completely switching off error correction, millions of new DNA sequences could be generated very quickly. This is how the immune system makes new immunoglobulins to neutralize an invading virus or bacterium. ${ }^{14}$ This process must be high level. The immune system as a whole must work out what will meet the new challenge. That cannot be done at the molecular level. The demons do not themselves know what they are doing. Nor do the hardworking cross-bridges within my muscles know why my fingers are typing this sentence. ${ }^{15}$

My view is consistent with Davies's exposition of molecular demons and what they do in the human bodyand with the conclusion that organisms are purposive. In a chapter entitled "Spooky Life and Quantum Demons," he writes that "life loves noise! Biology's demons harness thermal energy to create and to move." As for harnessing stochasticity, Davies asks, "Has nature overlooked to fill it? I don't think so."16

That, of course, makes two of us.

There remains the concept of information in biology. And about this concept, Davies is forthright:

While it is the case that biological information is instantiated in matter, it is not inherent in matter. Bits of information chart their own course inside living things. In so doing, they don't violate the laws of physics, but nor are they encapsulated by those laws: it is impossible to derive the laws of information from the known laws of physics. ${ }^{17}$

There is no need to defend this argument by means of the laws of physics. Consider a chessboard with the pieces arranged in any valid state of a game. No amount of physical analysis could reveal the rules of the game. Those rules are an invention of organisms, ourselves, and they could vary in almost limitless ways. As Davies writes, "The rules don't determine the outcome of the game, the players do." ${ }^{\prime 18} \mathrm{He}$ imagines a game he calls chess-plus. The players can change the rules during a game. In so doing, they will 
be following yet more rules, the rules for rule changing. Levels of meaning can be nested and this process can go on indefinitely.

The point goes over to an analysis of DNA. Davies compares DNA in two conditions, first when it is being used by the organism to make proteins, and second when it is being copied. In the first case, DNA is rather like the arrangement of chess pieces on the board. The organism makes sense of DNA as we make sense of a chess position. A particular sequence of nucleotides is meaningful inasmuch as the organism knows the mapping between DNA and its products. But neither the chess pieces nor the nucleotides enjoy anything like their meaning inherently. Meaning is irrelevant, after all, when DNA is copied. Before explorers chanced on the Rosetta stone, almost anyone could have faithfully copied any number of ancient Egyptian hieroglyphs without any understanding of their meaning.

Knowledge is necessarily relative to a knower. There was a time, now long gone, when it was thought that over $90 \%$ of the human genome was just junk with no meaning at all. The sequence was just the same when it was considered junk as it is today when we realize it is not. When Watson made his famous quip, "There are only molecules-everything else is sociology," he was perhaps wiser than he knew. ${ }^{19}$ Sociology is not physics, but social contexts are essential for understanding living organisms. Organisms signal to each other all the time, and unless someone can share in the culture of the local society, they will not necessarily understand what those signals mean.

Like the rest of the book, Davies's epilogue touches on deep questions about ourselves and the universe. One of these is whether life was inevitable. Some chemists are coming to the view that the assembly of peptides into polypeptides is not random. ${ }^{20}$ There may be a chemical bias toward the formation of living systems. Biology must lead thinkers to reconsider humanity and its place in the universe. At various points in the book, Davies hints at such implications. Summarizing what the revolution in evolution could mean, he ends with the statement, "It would be a universe in which we can truly feel at home."21 I, for one, find a universe that naturally gives rise to purposive and creative organisms more plausible, and more reassuring, than one that is completely purposeless. It is the universe as we experience it. The view that the universe is ruled by just blind chance, flies in the face of all that we experience as sentient, creative, and intentional beings. To believe this, we have to swallow the view that evolution, in creating the human nervous system, endowed it with an extraordinarily powerful illusion that forces us to act as though we have purpose, while really we only reflect the blind determination of our genes and other molecules.

We would then also have to live with an incoherent view of ourselves. We cannot, at one and the same time, deny that we have purpose and also write an article like this one, and even more so a complete book like The Demon in the Machine. Articles and books are necessarily written by purposive agents, not by random typewriting machines.

Denis Noble is Emeritus Professor of Cardiovascular Physiology at the University of Oxford.

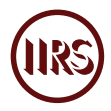

1. Erwin Schrödinger, What Is Life? (Oxford: Oxford University Press, 1943). This book was based on lectures that Schrödinger gave at the Dublin Institute of Advanced Studies after he left Germany.

2. Francis Crick, "Central Dogma of Molecular Biology," Nature 227 (1970): 561-63, doi:10.1038/227561a0. This article was an attempt to safeguard the original formulation in the 1950s. The problem was the discovery of reverse transcription of RNA into DNA. Crick's 1970 solution turned out to have been only a temporary delay of the deconstruction of the central dogma, since scientists now find that organisms themselves can edit their genomes.

3. I think that the first person to clearly identify this error in Schrödinger's work was Jean-Jacques Kupiec in his book The Origin of Individuals (Hackensack: World Scientific Publishing, 2009). The error was to assume that DNA copies itself in the same way as a crystal. It can't. A linear polymer does not have the 3D structure to be able to reproduce by forming a template for itself in that way. Kupiec also emphasized the role of natural selection within the cells of organisms.

4. Paul Davies, The Demon in the Machine (London: Penguin, 2019), 74.

5. George Ellis, Denis Noble, and Timothy O'Connor, “TopDown Causation: An Integrating Theme within and across the Sciences?" Interface Focus 2 (2012): 1-3, doi:10.1098/ rsfs.2011.0110. George Ellis, How Can Physics Underlie the Mind? (Berlin: Springer, 2016).

6. Denis Noble, "A Theory of Biological Relativity: No Privileged Level of Causation," Interface Focus 2 (2016): 55-64, doi:10.1098/rsfs.2011.0067.

7. This quotation is from a letter that Darwin sent to the explorer and natural historian Moritz Wagner in 1876. Quoted in Charles Darwin, Delphi Complete Works of Charles Darwin (Illustrated) (Hastings, Delphi Classics, 2015).

8. Davies, Demon in the Machine, 130. Kevin Laland, John Odling-Smee, and John Endler, "Niche Construction, Sources of Selection and Trait Coevolution," Interface Focus 7 (2017), doi:10.1098/rsfs.2016.0147.

9. Patrick Bateson, "The Active Role of Behaviour in Evolution," Biology and Philosophy 19, no. 2 (2004): 283-98, doi:10.1023/b:biph.0000024468.12161.83.

10. Davies, Demon in the Machine, 216.

11. Ellis, How Can Physics Underlie the Mind? 
12. Denis Noble, "Evolution Viewed from Physics, Physiology and Medicine," Interface Focus 7, no. 5 (2017), doi:10.1098/ rsfs.2016.0159.

13. Denis Noble, "Central Dogma or Central Debate?" Physiology 33 (2018): 246-49, doi:10.1152/physiol.00017.2018. This article conveys the essence of the misinterpretations of the central dogma in just three pages.

14. My co-author, Raymond Noble, and I are grateful to Richard Moxon for pointing out how remarkable this process of hypermutation is. E. Richard Moxon et al., "Adaptive Evolution of Highly Mutable Loci in Pathogenic Bacteria," Current Biology 4 (1994): 24-33, doi:10.1016/s0960-9822(00)00005-

1. E. Richard Moxon and David Thaler, "The Tinkerer's
Evolving Tool-Box," Nature 387 (1997): 659-62, doi:10.1038/ 42607.

15. Raymond Noble and Denis Noble, "Harnessing Stochasticity: How Do Organisms Make Choices?" Chaos 28, no. 106309 (2018), doi:10.1063/1.5039668.

16. Davies, Demon in the Machine, 165.

17. Davies, Demon in the Machine, 209.

18. Davies, Demon in the Machine, 212.

19. Book blurb by Vilayanur Ramachandran for Daniel Lieberman and Michael Long, Molecule of More (Dallas: BenBella Books, 2018).

20. Davies, Demon in the Machine, 215.

21. Davies, Demon in the Machine, 217.

Published on September 28, 2020 